\title{
Nonlinear response of a periodically driven damped two-state system
}

\author{
Milena Grifoni \\ Dipartimento di Fisica, Consorzio Interuniversitario Nazionale per la Fisica della Materia, \\ Università di Genova, I-16146 Genova, Italy \\ Maura Sassetti \\ Istituto di Fisica Ingegneria, Consorzio Interuniversitario Nazionale per la Fisica della Materia, \\ Università di Genova, I-16146 Genova, Italy \\ Jürgen Stockburger and Ulrich Weiss \\ Institut für Theoretische Physik, Universität Stuttgart, Pfaffenwaldring 57, W-7000 Stuttgart 80, Germany
}

(Received 18 March 1993)

\begin{abstract}
We study the nonlinear response of a periodically driven dissipative two-state system. The exact formal solution in the form of a series in the number of system transitions is derived. The series is summed in analytic form in the parameter region where incoherent transitions prevail. We also present the exact solution for the time evolution of the system in the entire region of temperatures and driving strength for a special value of the Ohmic viscosity. The destruction of quantum coherence by incoherent processes which are induced by the fluctuating and the driving forces is discussed on the basis of these solutions.

PACS number(s): 05.30. $-\mathrm{d}, 05.40 .+\mathrm{j}, 33.80 .-\mathrm{b}, 62.65 .+\mathrm{k}$
\end{abstract}

\section{INTRODUCTION}

Quantum-mechanical systems whose states are effectively confined to a two-dimensional Hilbert space are ubiquitous in physics and chemistry. For instance, imagine a particle resonating or fluctuating by quantummechanical tunneling between two different localized states. Such a system is frequently interacting with a heat bath allowing the system to enter a state of thermal equilibrium [1]. In many cases of practical interest it is adequate that the response of the environment be considered as linear. Then a thermal bath represented by a set of harmonic oscillators with a bilinear coupling in the system's and reservoir's coordinates captures the essential physics [2]. Since the two-state or two-level system (TLS) is like a spin, the corresponding model has become known in the literature as the spin-boson model [3]. Within the vast literature, the thermodynamics and dynamics of this model have been discussed in the context of quantum optics, chemical physics (e.g., electron transfer reactions), amorphous materials, and polaron theory, to mention a few.

In this paper, we study the influence of periodic driving on the dynamics of a damped two-state system. This model is simple enough that it is at least partly tractable by analytic methods. Nevertheless, it shows many of the characteristic features of more realistic systems. The model is versatile to describe, e.g., ac-driven superconducting quantum interference devices (SQUID's) [4, 5], laser-induced isomerization of bistable molecules [6], laser-induced localization of electrons in semiconductor double-well quantum structures [7], and paraelectric resonances [8].

At low temperatures, the essential dynamics of such systems corresponds to a Hilbert space with dimension two. In many circumstances, the amplitude of the driving field is so large that the linear response approximation is not valid, and a more profound treatment is required.

A main theme of driven damped bistable systems is the reduction of the coherent tunneling motion by incoherent processes which are induced by the variation of the tunneling splitting by fluctuating and driving forces. The frictional influences can lead to qualitative changes in the behaviors. Quite generally, the stochastic force results in a reduction of the tunneling process [1-3], and may even lead to a fluctuation-induced phase transition to self-trapping at zero temperature [9]. Quenching of quantum tunneling may also be induced by external periodic driving in some regions of the parameter space $[10$, 11].

Our study is also partly motivated by recent experiments on acoustic properties of amorphous metals [12, 13]. These experiments showed strong deviations from the expected logarithmic temperature dependence of the sound velocity and a drastic decrease of the internal friction below about $30 \mathrm{mK}$. The standard phenomenological tunneling model [14] can only partially explain [15] the observed low-frequency properties of the investigated amorphous materials. The anomalies have been tentatively interpreted in terms of a low-energy cutoff in the distribution of tunneling splittings leading to an upper bound of relaxation times induced by the conduction electrons $[12,16]$. However, further extensions of the theoretical description are imperative. Since most of the data reported on acoustic properties in Ref. [13] show amplitude-dependent effects, the strain fields in the vibrating reed or vibrating wire experiments were sufficiently strong that the linear response regime is exceeded. This strengthened our interest in the study of bias-amplitude dependent effects in driven damped two- 
state systems.

The outline of the paper is as follows. In Sec. II we formulate the problem, derive the exact formal solution, and give a brief discussion of the linear response regime. Section III is devoted to the nonlinear response in the regime of incoherent tunneling. We present the solution for the steady state for different shapes of the driving force. In Sec. IV we solve the dynamics exactly in analytic form for any temperature and driving parameters for a special value of the Ohmic damping strength. This particular case shows many of the characteristic features of a driven damped two-state system. Finally, in Sec. V we summarize the findings and draw our conclusions.

\section{DYNAMICS OF THE DRIVEN SPIN-BOSON SYSTEM}

Many physical and chemical systems, among them those mentioned in the Introduction, can be described by a generalized coordinate with which is associated an effective potential energy function with two separate minima. We characterize the double well by the barrier height $V_{0}$, by the separation $\hbar \omega_{0}$ of the first excited state from the ground state in either well, and by an intrinsic "detuning" energy $\hbar \epsilon_{0}$ between the ground states in the two wells. Then in the parameter regime $V_{0} \gg \hbar \omega_{0} \gg \hbar \epsilon_{0}, k_{B} T$, the system will be effectively restricted to the two-dimensional Hilbert space spanned by the two ground states. Taking into account the possibility of tunneling between the two wells, the TLS is then governed by the pseudospin Hamiltonian

$$
H_{\mathrm{TLS}}=-\left(\frac{\hbar \Delta}{2}\right) \sigma_{x}-\left(\frac{\hbar \epsilon_{0}}{2}\right) \sigma_{z} .
$$

Here the $\sigma$ 's are Pauli matrices, and the basis is chosen such that the states $|r\rangle$ (right) and $|l\rangle$ (left) are eigenstates of $\sigma_{z}$ with eigenvalues +1 and -1 , respectively. The interaction energy $\hbar \Delta$ is the energy splitting of a symmetric TLS due to tunneling.

We shall consider linear couplings to the heat bath that are sensitive to the value of $\sigma_{z}$. For example, a dipole-local-field coupling provides a simple physical model for this type of coupling. To be definite, we choose $H_{I}=-q \sum_{\alpha} c_{\alpha} x_{\alpha}$ where $q=\sigma_{z} a / 2$ with $a$ being a characteristic length (usually the spatial distance of the two localized states), and $x_{\alpha}$ is a bath coordinate. Then, by representing the reservoir by a set of harmonic oscillators, we end up with the spin-boson Hamiltonian [3]

$$
H_{\mathrm{SB}}=H_{\mathrm{TLS}}+\frac{1}{2} \sum_{\alpha}\left(\frac{p_{\alpha}^{2}}{m_{\alpha}}+m_{\alpha} \omega_{\alpha}^{2} x_{\alpha}^{2}-c_{\alpha} x_{\alpha} a \sigma_{z}\right) \text {. }
$$

Despite its apparent simplicity, the spin-boson model cannot be solved exactly by any known method.

Finally, we add an interaction term in which the bias energy is modulated by an externally applied timedependent force $\hbar \epsilon(t) / a$. Then, the Hamiltonian of the driven spin-boson system is

$$
H=H_{\mathrm{SB}}-\hbar \epsilon(t) \sigma_{z} / 2 .
$$

Hence, the two-state system with coupling proportional to $\sigma_{x}$ propagates under the influence of three biasing forces: an intrinsic constant force $\hbar \epsilon_{0} / a$, a timedependent (externally applied) force $\hbar \epsilon(t) / a$, and a fluctuating force $\xi(t)=\sum_{\alpha} c_{\alpha} x_{\alpha}(t)$. The quantity $\hbar \epsilon_{0}$ is the intrinsic asymmetry energy of the double-well potential of the extended system, and $\hbar \hat{\epsilon}(t)$ is the time-dependent asymmetry energy due to the external driving force. For a harmonic bath, the fluctuating force obeys Gaussian statistics, and is fully characterized by $\langle\xi(t)\rangle_{\beta}=0$ and by the force autocorrelation function in thermal equilibrium $[1,3]$

$$
\begin{aligned}
\langle\xi(t) \xi(0)\rangle_{\beta} & =\hbar \sum_{\alpha} \frac{c_{\alpha}^{2}}{2 m_{\alpha} \omega_{\alpha}} \frac{\cosh \left[\omega_{\alpha}(\hbar \beta / 2-i t)\right]}{\sinh \left[\omega_{\alpha} \hbar \beta / 2\right]} \\
& =\frac{\hbar}{\pi} \int_{0}^{\infty} d \omega J(\omega) \frac{\cosh [\omega(\hbar \beta / 2-i t)]}{\sinh (\omega \hbar \beta / 2)}
\end{aligned}
$$

where $\beta \equiv 1 / k_{B} T$, and where in the second form we have introduced the spectral function

$$
J(\omega)=\frac{\pi}{2} \sum_{\alpha} \frac{c_{\alpha}^{2}}{m_{\alpha} \omega_{\alpha}} \delta\left(\omega-\omega_{\alpha}\right)
$$

of the environmental coupling. Since we wish the environment to constitute a proper heat bath, we shall consider $J(\omega)$ as continuous henceforth. In the classical description, the spectral density $J(\omega)$ is related to the frequency-dependent friction [2]. Here we will assume that at the relevant frequencies the friction is Ohmic or independent of frequency. This case is described by a spectral density of the form [1]

$$
J(\omega)=\eta \omega e^{-\omega / \omega_{c}}=\left(2 \pi \hbar \alpha / a^{2}\right) \omega e^{-\omega / \omega_{c}} .
$$

Here, $\eta$ is the phenomenological Ohmic viscosity coefficient, while $\alpha$ is the standard dimensionless coupling constant [3], and we made the specific choice of an exponential cutoff. In what follows, we shall assume that the cutoff frequency $\omega_{c}$ in the environmental modes is the highest frequency of the problem. At low temperatures, the very low-frequency dependence of $J(\omega)$ is crucial for the qualitative physics $[1,3]$. Ohmic dissipation is widespread in physical and chemical situations. This has been shown, e.g., by molecular-dynamical simulations. In metallic glasses at low temperatures, the TLS's are affected by Ohmic friction due to excitation of electronhole pairs near the Fermi surface $[14,17]$.

\section{A. Exact formal solution of the system dynamics}

The dynamical quantity we are interested in is the expectation value $\left\langle\sigma_{z}(t)\right\rangle$ of the TLS for the case that the TLS-plus-reservoir complex is described at time zero by a density matrix in factorized form. To be definite, we shall assume that the TLS is initially in the state $|r\rangle$ while the bath is in thermal equilibrium. Then, the expectation value of $\sigma_{z}$ takes the form

$\left\langle\sigma_{z}(t)\right\rangle \equiv P(t)=P(+, t ;+, 0)-P(-, t ;+, 0)$,

where $P\left(\sigma, t ; \sigma^{\prime}, t^{\prime}\right)$ is the joint probability for finding the 
system in the state $\sigma$ at time $t$ when it was released without uncertainty from the state $\sigma^{\prime}$ at time $t^{\prime}$.

Actually, we are only interested in the steady-state response for periodic driving reached at large times, and not in transient effects. Now, in this limit, the contribution to $P(t)$ which is symmetric under the inversion $\mathcal{E}(t) \rightarrow-\mathcal{E}(t)$, where $\mathcal{E}(t) \equiv \epsilon_{0}+\epsilon(t)$, is damped away. Therefore we can restrict the attention to the contribution of $P(t)$ which is antisymmetric under the symmetry transformation $\mathcal{E}(t) \rightarrow-\mathcal{E}(t)$. This part is given by

$$
P^{(a)}(t)=\frac{1}{2} \sum_{\sigma, \sigma^{\prime}} \sigma P\left(\sigma, t ; \sigma^{\prime}, 0\right)
$$

To formulate the evolution of $P^{(a)}(t)$, it is convenient to use the real-time influence functional method developed by Feynman and Vernon for a product form of the initial state of the composite system [18]. Formally, the joint probability is given by the double path integral expression

$P\left(\sigma, t ; \sigma^{\prime}, 0\right)=\int \mathcal{D} q \int \mathcal{D} q^{\prime} \mathcal{A}[q] \mathcal{B}[q] \mathcal{A}^{*}\left[q^{\prime}\right] \mathcal{B}^{*}\left[q^{\prime}\right] \mathcal{F}\left[q, q^{\prime}\right]$

in which the sum is over all real-time paths $q\left(t^{\prime}\right), q^{\prime}\left(t^{\prime}\right)$ with constraints $q(0)=q^{\prime}(0)=\sigma^{\prime} a / 2, q(t)=q^{\prime}(t)=$ $\sigma a / 2$. For the TLS we have $q\left(t^{\prime}\right)=a \sigma\left(t^{\prime}\right) / 2$ and $q^{\prime}\left(t^{\prime}\right)=a \sigma^{\prime}\left(t^{\prime}\right) / 2$, where both $\sigma\left(t^{\prime}\right)$ and $\sigma^{\prime}\left(t^{\prime}\right)$ jump back and forth between the values +1 and -1 . The quantity $\mathcal{A}[q]$ is the probability amplitude of the TLS to follow the path $q\left(t^{\prime}\right)$ in the absence of biasing and fluctuating forces. The factor

$$
\mathcal{B}[q]=\exp \left\{i \int_{0}^{t} d t^{\prime}\left[\epsilon_{0}+\epsilon\left(t^{\prime}\right)\right] q\left(t^{\prime}\right) / a\right\}
$$

incorporates the effect of the biasing force, and $\mathcal{F}\left[q, q^{\prime}\right]$ is the Feynman-Vernon influence functional which describes the influences of the fluctuating force. For Gaussian statistics, it takes the form

$$
\begin{aligned}
\mathcal{F}\left[q, q^{\prime}\right]= & \exp \left\{-\int_{0}^{t} d t^{\prime} \int_{0}^{t^{\prime}} d t^{\prime \prime}\left[q\left(t^{\prime}\right)-q^{\prime}\left(t^{\prime}\right)\right]\right. \\
& \left.\times\left[K\left(t^{\prime}-t^{\prime \prime}\right) q\left(t^{\prime \prime}\right)-K^{*}\left(t^{\prime}-t^{\prime \prime}\right) q^{\prime}\left(t^{\prime \prime}\right)\right]\right\}
\end{aligned}
$$

where $K(t) \equiv\langle\xi(t) \xi(0)\rangle_{\beta}$ is the force autocorrelation function given in (2.4). Finally, $\int \mathcal{D} q$ symbolically means summation in function space over all paths with the end points held fixed.

The exact formal expression for $P^{(a)}(t)$ has been derived in Refs. $[3,1]$. We now give the main formulas relevant for our purposes and generalized to the case of a time-dependent bias.

In real-time studies, it is convenient to introduce the antisymmetric and symmetric coordinates

$$
\xi(t)=\frac{1}{a}\left[q(t)-q^{\prime}(t)\right], \quad \eta(t)=\frac{1}{a}\left[q(t)+q^{\prime}(t)\right] .
$$

Now, a two-state system starting out from a diagonal state of the density matrix is again in a diagonal state after any even number of transitions, and in an off-diagonal state after any odd number of transitions. Since the flips are sudden, a general path with $2 n$ transitions at flip times $t_{j}(j=1,2, \ldots, 2 n)$ within the interval $0<t^{\prime}<t$ is then parametrized by

$$
\begin{aligned}
& \xi_{n}\left(t^{\prime}\right)=\sum_{j=1}^{n} \xi_{j}\left(\Theta\left(t^{\prime}-t_{2 j-1}\right)-\Theta\left(t^{\prime}-t_{2 j}\right)\right), \\
& \eta_{n}\left(t^{\prime}\right)=\sum_{j=0}^{n} \eta_{j}\left(\Theta\left(t^{\prime}-t_{2 j}\right)-\Theta\left(t^{\prime}-t_{2 j+1}\right)\right),
\end{aligned}
$$

where $t_{0} \equiv 0, t_{2 n+1} \equiv t$, and where $\Theta(t)$ is the unit step function. The labels $\xi_{j}= \pm 1$ and $\eta_{j}= \pm 1$ mark the two off-diagonal and diagonal states of the density matrix, respectively. The periods $t_{2 j}<t^{\prime}<t_{2 j+1}$, in which the system is in a diagonal state, are usually referred to as sojourns, and the periods $t_{2 j-1}<t^{\prime}<t_{2 j}$, in which the system stays off diagonal, are called blips (cf. Ref. [3]). In this parametrization, the sum over histories of paths is represented (1) by the sum over any number $n$ of flip pairs, (2) by the time-ordered integrations over the $2 n$ flip times $\left\{t_{j}\right\}$ within the given interval, and (3) by the sum over all arrangements $\left\{\xi_{j}\right\}$ and $\left\{\eta_{j}\right\}$ of the possible values \pm 1 of the individual $\xi_{j}$ and $\eta_{j}$. Introducing for the time integrals the compact notation

$$
\int_{0}^{t} \mathcal{D}_{n}\left\{t_{j}\right\} \cdots \equiv \int_{0}^{t} d t_{2 n} \int_{0}^{t_{2 n}} d t_{2 n-1} \cdots \int_{0}^{t_{2}} d t_{1} \cdots,
$$

the path summation takes the form

$$
\int \mathcal{D} q \int \mathcal{D} q^{\prime} \cdots \quad \Longrightarrow \quad \sum_{n=0}^{\infty} \int_{0}^{t} \mathcal{D}_{n}\left\{t_{j}\right\} \sum_{\left\{\xi_{j}\right\}} \sum_{\left\{\eta_{j}\right\}} \cdots
$$

The amplitude per unit time to switch from a diagonal to an off-diagonal state, or vice versa, is $\pm i \Delta / 2$, and the amplitude to stay in a sojourn is unity. Further, the product of amplitudes for $n$ intermediate blips depends on the bias and is $\exp \left(i \Phi_{n}\right)$. The phase $\Phi_{n}$ receives contributions from the static and from the time-dependent strain field. We have

$$
\begin{aligned}
\Phi_{n} & =\Phi_{n}^{(0)}+\Phi_{n}^{(1)}, \\
\Phi_{n}^{(0)} & =\epsilon_{0} \sum_{j=1}^{n} \xi_{j}\left(t_{2 j}-t_{2 j-1}\right), \\
\Phi_{n}^{(1)} & =\sum_{j=1}^{n} \xi_{j}\left[g\left(t_{2 j}\right)-g\left(t_{2 j-1}\right)\right],
\end{aligned}
$$

where the function $g(t)$ is given by the expression

$$
g(t)=\int_{0}^{t} d t^{\prime} \epsilon\left(t^{\prime}\right)
$$


Finally, the influence functional for $2 n$ transitions between the states of the density matrix reads

$$
\begin{aligned}
& \mathcal{F}_{n}=G_{n}\left(\left\{\xi_{j}\right\}\right) H_{n}\left(\left\{\xi_{j}\right\},\left\{\eta_{j}\right\}\right) \\
& G_{n}=\exp \left(-\sum_{j=1}^{n} S_{2 j, 2 j-1}-\sum_{j=2}^{n} \sum_{k=1}^{j-1} \xi_{j} \xi_{k} \Lambda_{j, k}\right), \\
& H_{n}=\exp \left(i \sum_{j=1}^{n} \xi_{j} \sum_{k=0}^{j-1} \eta_{k} X_{j, k}\right) .
\end{aligned}
$$

The function $G_{n}$ describes the interactions of the $n$ blips. Here we broke them up into the self-interactions and the interactions between blip pairs. The factor $H_{n}$ includes the interactions of each of the blips with the respective preceding sojourns. The interactions are given by the expressions

$$
\begin{aligned}
S_{j, k} & =S\left(t_{j}-t_{k}\right), \quad R_{j, k}=R\left(t_{j}-t_{k}\right) \\
\Lambda_{j, k} & =S_{2 j, 2 k-1}+S_{2 j-1,2 k}-S_{2 j, 2 k}-S_{2 j-1,2 k-1} \\
X_{j, k} & =R_{2 j, 2 k+1}+R_{2 j-1,2 k}-R_{2 j, 2 k}-R_{2 j-1,2 k+1}
\end{aligned}
$$

where the functions $S(\tau)$ and $R(\tau)$ are related to the real and imaginary part of the second integral of the force autocorrelation function, respectively. We have

$$
\begin{aligned}
& S(\tau)=\frac{a^{2}}{\pi \hbar} \int_{0}^{\infty} d \omega \frac{J(\omega)}{\omega^{2}}(1-\cos \omega \tau) \operatorname{coth}\left(\frac{\hbar \beta \omega}{2}\right), \\
& R(\tau)=\frac{a^{2}}{\pi \hbar} \int_{0}^{\infty} d \omega \frac{J(\omega)}{\omega^{2}} \sin \omega \tau .
\end{aligned}
$$

In the Ohmic case and for times $\tau \gg 1 / \omega_{c}$, the complex interaction is given by

$$
S(\tau)+i R(\tau)=\alpha\left\{2 \ln \left[\frac{\hbar \beta \omega_{c}}{\pi} \sinh \left(\frac{\pi \tau}{\hbar \beta}\right)\right]+i \pi\right\}
$$

Collecting all factors, the joint probability takes the form of the series expression

$$
\begin{aligned}
P\left(\sigma, t ; \sigma^{\prime}, 0\right)=\delta_{\sigma, \sigma^{\prime}}+ & \sigma \sigma^{\prime} \sum_{n=1}^{\infty}\left(-\frac{\Delta^{2}}{4}\right)^{n} \\
& \times \int_{0}^{t} \mathcal{D}_{n}\left\{t_{j}\right\} \sum_{\left\{\xi_{j}\right\}} G_{n} e^{i \Phi_{n}} \sum_{\left\{\eta_{j}\right\}^{\prime}} H_{n},
\end{aligned}
$$

where the prime in $\left\{\eta_{j}\right\}^{\prime}$ indicates that the outer sojourns are chosen according to the boundary conditions, i.e., $\eta_{0}=\sigma^{\prime}$ and $\eta_{n}=\sigma$. The expression (2.20) is in the form of an expansion in even numbers of transitions between the four states of the density matrix. The transitions occur at times $\left\{t_{j}\right\}$, and the sum over $\left\{\xi_{j}\right\}$ and $\left\{\eta_{j}\right\}^{\prime}$ takes into account all possibilities of intervening states in a given order $\Delta^{2 n}$. It is now straightforward to perform the summation over the inner $\eta_{j}$. The resulting expression can be simplified further if we insert for the interaction the form (2.19) of the Ohmic case. We finally obtain

$$
\begin{aligned}
P^{(a)}(t)= & -\sum_{n=1}^{\infty}\left(-\frac{\Delta^{2} \cos (\pi \alpha)}{2}\right)^{n} \\
& \times \int_{0}^{t} \mathcal{D}_{n}\left\{t_{j}\right\} \sum_{\left\{\xi_{j}\right\}} \tan \left(\pi \alpha \xi_{1}\right) G_{n} \sin \Phi_{n}
\end{aligned}
$$

The result (2.21) represents the exact formal solution for the evolution of $P^{(a)}(t)$ under time-dependent external strains in the Ohmic case. It forms the basis of our subsequent work.

To study the frequency-dependent response, it is convenient to turn to the Laplace transform $\widehat{P}^{(a)}(\lambda)=$ $\int_{0}^{\infty} d t P^{(a)}(t) e^{-\lambda t}$. To this, we introduce the blip lengths $\tau_{j}$ and sojourn lengths $s_{j}$, respectively,

$\tau_{j}=t_{2 j}-t_{2 j-1}, \quad s_{j}=t_{2 j+1}-t_{2 j} \quad(j=1,2, \ldots, n)$,

where $s_{0}=t_{1}$. With the abbreviation

$$
\begin{aligned}
\int_{0}^{\infty} \widetilde{\mathcal{D}}_{n}(\lambda) \cdots \equiv \prod_{i=1}^{n} & \left(\int_{0}^{\infty} d \tau_{i} e^{-\lambda \tau_{i}}\right) \\
& \times \prod_{j=1}^{n-1}\left(\int_{0}^{\infty} d s_{j} e^{-\lambda s_{j}}\right) \cdots
\end{aligned}
$$

the Laplace transform of $P^{(a)}(t)$ takes the form

$$
\begin{aligned}
\widehat{P}^{(a)}(\lambda)= & -\frac{1}{\lambda} \sum_{n=1}^{\infty}\left(-\frac{\Delta^{2} \cos \pi \alpha}{2}\right)^{n} \\
& \times \int_{0}^{\infty} \widetilde{\mathcal{D}}_{n}(\lambda) \int_{0}^{\infty} d s_{0} e^{-\lambda s_{0}} \\
& \times \sum_{\left\{\xi_{j}\right\}} \tan \left(\pi \alpha \xi_{1}\right) G_{n} \sin \Phi_{n} .
\end{aligned}
$$

For later convenience, we have kept explicitly in our notation the integration over the initial sojourn length $s_{0}$. In terms of the blip and sojourn lengths, the phases $\Phi_{n}^{(0)}$ and $\Phi_{n}^{(1)}$ read

$$
\begin{aligned}
\Phi_{n}^{(0)}=\epsilon_{0} \sum_{j=1}^{n} \xi_{j} \tau_{j}, \\
\Phi_{n}^{(1)}=\sum_{j=1}^{n} \xi_{j}\left\{g\left(s_{0}+\tau_{j}+\sum_{l=1}^{j-1}\left(\tau_{l}+s_{l}\right)\right)\right. \\
\left.-g\left(s_{0}+\sum_{l=1}^{j-1}\left(\tau_{l}+s_{l}\right)\right)\right\} .
\end{aligned}
$$

Before discussing the periodically driven system in the general case, we now first draw the attention to the linear response regime. 


\section{B. Linear response \\ to a time-periodic harmonic force}

Consider a two-state system driven periodically with frequency $\omega$,

$$
\epsilon(t)=\hat{\epsilon} \cos (\omega t-\varphi)
$$

and linearize Eq. (2.24) in $\Phi_{n}^{(1)}$. It is then easy to perform the $s_{0}$ integration. The result is

$\widehat{P}^{(a, l)}(\lambda)=\hat{\epsilon}\left[\frac{\mathcal{F}(\lambda, \omega)}{\lambda-i \omega} e^{-i \varphi}+\frac{\mathcal{F}(\lambda,-\omega)}{\lambda+i \omega} e^{i \varphi}\right]$

where the function $\mathcal{F}(\lambda, \omega)$ depends only on the intrinsic properties of the system,

$$
\begin{aligned}
\mathcal{F}(\lambda, \omega)= & \frac{i}{2 \omega \lambda} \sum_{n=1}^{\infty}\left(-\frac{\Delta^{2} \cos \pi \alpha}{2}\right)^{n} \\
& \times \int_{0}^{\infty} \widetilde{\mathcal{D}}_{n}(\lambda) \sum_{\left\{\xi_{k}\right\}} \tan \left(\pi \alpha \xi_{1}\right) G_{n} \cos \Phi_{n}^{(0)} \\
& \times \sum_{j=1}^{n} \xi_{j} e^{i \omega \sum_{l=1}^{j-1}\left(\tau_{l}+s_{l}\right)}\left(e^{i \omega \tau_{j}}-1\right)
\end{aligned}
$$

The transform $\widehat{P}^{(a, l)}(\lambda)$ can then be inverted to obtain $P^{(a, l)}(t)$,

$$
P^{(a, l)}(t)=\frac{1}{2 \pi i} \int_{\mathcal{C}} d \lambda \widehat{P}^{(a, l)}(\lambda) e^{\lambda t}
$$

where $\mathcal{C}$ is the standard Bromwich contour lying to the right of all singularities of $\widehat{P}^{(a, l)}(\lambda)$. Here, $P^{(a, l)}(t)$ is made up of two different contributions. The one arises from the poles of the function $\mathcal{F}(\lambda, \omega)$, the other is due to the additional poles at $\lambda= \pm i \omega$. The poles in the first case depend on the parameters of the system, and for $\alpha \neq 0$ they are lying entirely in the left-hand half plane. Hence, all their contributions to $P^{(a, l)}(t)$ will decay exponentially. Therefore they only describe the transient response of the system. It is just the second sort of poles which gives the steady-state behavior of the system reached at large times. We find

$$
\begin{aligned}
P^{(\mathrm{st}, l)}(t)= & \hat{\epsilon}\left[\mathcal{F}(\lambda=i \omega, \omega) e^{i(\omega t-\varphi)}\right. \\
& \left.+\mathcal{F}(\lambda=-i \omega,-\omega) e^{-i(\omega t-\varphi)}\right]
\end{aligned}
$$

From this we see that a phase shift in the driving force simply gives an identical phase shift in $P^{(s t, l)}(t)$. Henceforth, we shall set $\varphi=0$.

Using Kubo's linear response theory, one finds that $P^{(\text {st, } l)}(t)$ is related to the linear dynamical susceptibility $\widetilde{\chi}(\omega)$ by

$$
P^{(\mathrm{st}, l)}(t)=\hbar \hat{\epsilon}\left[\tilde{\chi}(-\omega) e^{i \omega t}+\tilde{\chi}(\omega) e^{-i \omega t}\right]
$$

Comparing now (2.30) with (2.31), and using (2.28), we get

$$
\begin{aligned}
\widetilde{\chi}(\omega)= & \frac{1}{\hbar} \mathcal{F}(\lambda=-i \omega,-\omega) \\
= & \frac{1}{2 \hbar \omega^{2}} \sum_{n=1}^{\infty}\left(-\frac{\Delta^{2} \cos \pi \alpha}{2}\right)^{n} \\
& \times \int_{0}^{\infty} \widetilde{\mathcal{D}}_{n}(-i \omega) \sum_{\left\{\xi_{j}\right\}} \tan \left(\pi \alpha \xi_{1}\right) G_{n} \cos \Phi_{n}^{(0)} \\
& \times \sum_{k=1}^{n} \xi_{k} e^{-i \omega \sum_{l=1}^{k-1}\left(\tau_{l}+s_{l}\right)}\left(e^{-i \omega \tau_{k}}-1\right)
\end{aligned}
$$

The dynamical susceptibility $\tilde{\chi}(\omega)$ describes the system's response to a weak external perturbation. In spectroscopy experiments with electromagnetic or elastic waves, the real and imaginary parts of $\widetilde{\chi}(\omega)$ determine the dispersive and absorptive properties of the TLS, respectively. It is interesting to see whether the expression (2.32) coincides with the dynamical susceptibility calculated as the Fourier transform of the retarded susceptibility

$$
\chi(t)=\frac{i}{\hbar a^{2}} \theta(t)\left\langle[q(t), q(0)]_{-}\right\rangle_{\beta},
$$

where $q(t)=a \sigma_{z}(t) / 2$. Here, the driving force $\hbar \epsilon(t) / a$ is disregarded, and \langle\rangle$_{\beta}$ means thermal average with respect to the system-plus-reservoir Hamiltonian.

There are two important reasons for checking the equivalence of the two different approaches. First, if verified, there would be in fact two different starting points for a computation of the dynamical susceptibility. In the former approach, we assumed a product initial state of the system-plus-reservoir complex for $P^{(a, l)}(t)$, while if we had agreed to calculate $\tilde{\chi}(\omega)$ from $\chi(t)$, we had to consider a correlated thermal initial state. Second, and more importantly, it is only the first approach which is easily generalizable to the nonlinear response regime.

We now confirm the equivalence of the two different approaches. From this we shall deduce our confidence in the first method, which we shall apply in the following sections to the study of the nonlinear response.

When the coupled system-plus-reservoir complex is ergodic, the system will relax towards thermal equilibrium independently of the chosen initial state. Thus we may choose for simplicity at $t=t_{0}$ a product state, and the particle will have equilibrated with the bath at time $t=0_{-}$if we shift $t_{0}$ to the infinite past. Performing now at time $t=0$ a measurement of the observable in question, we just have the kind of a correlated thermal initial state. When the same observable is measured again at time $t>0$, we gain information about the equilibrium correlation function of this quantity. Regarding the correlation function $\left\langle\sigma_{z}(t) \sigma_{z}(0)\right\rangle_{\beta}$, we have to study the propagating function $J\left(\xi, \eta, t ; \xi^{\prime}, \eta^{\prime}, 0 ; \xi^{\prime \prime}, \eta^{\prime \prime}, t_{0}\right)$ in the limit $t_{0} \rightarrow-\infty$ for $\xi=0, \eta= \pm 1$ and $\xi^{\prime \prime}=0, \eta^{\prime \prime}= \pm 1$. Since we may neglect system-bath correlations in the infinite past, the environmental influences can again be described by the standard Feynman-Vernon influence functional. Now, the correlations due to the thermal initial state at $t=0$ are in the interactions between the negative- and positive-time branches conveyed by the influence functional. 
In an earlier work [19], two of the authors have used this method to compute the symmetrized correlation function. It is straightforward to adjust the procedure just outlined to the computation of the retarded susceptibility. The main difference between the retarded susceptibility and the symmetrized correlation function is that in the former case, due to the commutator in (2.33), the system must be at time $t=0$ in an off-diagonal state of the density matrix, i.e., in a blip state $\xi^{\prime}= \pm 1, \eta^{\prime}=0$. As a result, $\chi(t)$ receives contributions from paths with any odd number of transitions at negative and at positive times. Summing over all paths, we arrive at the exact formal expression

$$
\begin{aligned}
\chi(t)= & -\frac{1}{2 \hbar} \theta(t) \sum_{n=1}^{\infty}\left(-\frac{\Delta^{2} \cos \pi \alpha}{2}\right)^{n} \\
& \times \sum_{k=1}^{n} \int_{0}^{t} d t_{2 n} \cdots \int_{0}^{t_{2 k+1}} d t_{2 k} \\
& \times \int_{-\infty}^{0} d t_{2 k-1} \cdots \int_{-\infty}^{t_{2}} d t_{1} \\
& \times \sum_{\left\{\xi_{j}\right\}} \xi_{k} \tan \left(\pi \alpha \xi_{1}\right) G_{n} \cos \Phi_{n}^{(0)}
\end{aligned}
$$

Switching to the Laplace transform, it is again convenient to introduce the blip and sojourn lengths $\left\{\tau_{j}\right\}$ and $\left\{s_{j}\right\}$, respectively. Doing this, a special role is played by the blip length $\tau_{k}=\tau_{k}^{\prime}+\tau_{k}^{\prime \prime}$, where $\tau_{k}^{\prime \prime}=-t_{2 k-1}$ and $t_{k}^{\prime}=t_{2 k}$ represent the periods spent in the blip state $\xi_{k}$ at negative and positive times, respectively. Since the integrand $f_{n} \equiv$ $G_{n} \cos \Phi_{n}^{(0)}$ depends only on $\tau_{k}$, and not on $\tau_{k}^{\prime}$ and $\tau_{k}^{\prime \prime}$ separately, the occurring integrals over $\tau_{k}^{\prime}$ and $\tau_{k}^{\prime \prime}$ may be transformed according to

$$
\begin{aligned}
\int_{0}^{\infty} d \tau_{k}^{\prime} \int_{0}^{\infty} d \tau_{k}^{\prime \prime} f_{n}\left(\tau_{k}^{\prime}+\tau_{k}^{\prime \prime}\right) e^{-\lambda \tau_{k}^{\prime}} \\
=\frac{1}{\lambda} \int_{0}^{\infty} d \tau_{k} f_{n}\left(\tau_{k}\right)\left(1-e^{-\lambda \tau_{k}}\right) .
\end{aligned}
$$

Thus we find

$$
\begin{aligned}
\widehat{\chi}(\lambda)= & -\frac{1}{2 \hbar \lambda^{2}} \sum_{n=1}^{\infty}\left(-\frac{\Delta^{2} \cos \pi \alpha}{2}\right)^{n} \\
& \times \int_{0}^{\infty} \widetilde{\mathcal{D}}_{n}(\lambda) \sum_{\left\{\xi_{j}\right\}} \tan \left(\pi \alpha \xi_{1}\right) G_{n} \cos \Phi_{n}^{(0)} \\
& \times \sum_{k=1}^{n} \xi_{k} e^{\lambda \sum_{l=1}^{k-1}\left(\tau_{l}+s_{l}\right)}\left(e^{+\lambda \tau_{k}}-1\right)
\end{aligned}
$$

Recalling now that $\tilde{\chi}(\omega)=\widehat{\chi}(\lambda=-i \omega)$, we immediately see that the resulting $\widetilde{\chi}(\omega)$ agrees with the previous findings (2.32). We have therefore proved that the two methods for calculating the linear dynamical susceptibility are indeed equivalent.

\section{THE INCOHERENT REGIME}

Despite its complicated appearance, the expression (2.21) for $P^{(a)}(t)$ can be summed in certain limits by analytic methods. In this section, we analyze the behavior of $P^{(a)}(t)$ at long times in the regime where the driving frequency $\omega$ matches the time scale of incoherent relaxation. We will choose

$$
\omega \ll 2 \pi \alpha / \hbar \beta
$$

as a parameter range to start with. Our results will show that the effects of incoherent relaxation will indeed be found mainly in this frequency range. For constant bias, the main difficulty in an analytic resummation of $P^{(a)}(t)$ originates from the interactions $\Lambda_{j, k}$ between blip pairs contained in the influence factor $G_{n}$. We shall refer to them as interblip correlations. With external driving, the situation is even worse than in the static case because we have to deal with the correlations given by the phase $\Phi_{n}^{(1)}$ in addition to the interactions $\Lambda_{j, k}$ due to dissipation.

First, we observe that the intrablip interactions $S_{2 j, 2 j-1}$ in the factor $G_{n}$ lead for the Ohmic form (2.19) to an exponential cutoff $\exp \left(-2 \alpha \pi \tau_{j} / \hbar \beta\right)$ on the blip length $\tau_{j}$, while long sojourn lengths are not suppressed by a corresponding factor. Thus for driving frequencies in the range (3.1), we may linearize the phase $\Phi_{n}^{(1)}$ in (2.25) with respect to all the blip lengths $\tau_{j}$ giving the total phase the simpler form

$$
\Phi_{n}=\sum_{j=1}^{n} \xi_{j}\left[\epsilon_{0}+\epsilon\left(\sum_{l=0}^{j-1} s_{l}\right)\right] \tau_{j} .
$$

Now, the external driving force behaves as an effective time-dependent bias which still depends on the preceding sojourn lengths.

Second, we will study the environmental influences within the noninteracting-blip approximation (NIBA) in which the interblip correlations $\Lambda_{j, k}$ are neglected. The self-consistency of this approximation is discussed in Ref. [3]. For strong damping $\alpha>1$, the NIBA is justified in the entire parameter region, while for weak damping $\alpha \ll 1$ it is valid in the region

$$
k_{B} T \gg \hbar \Delta \quad \text { or } \quad\left|\epsilon_{0}+\epsilon(t)\right| \gg \Delta .
$$

Inserting (3.2) in (2.24) and setting $\Lambda_{j, k}=0$, the series expression for $\widehat{P}^{(a)}(\lambda)$ can be rewritten as

$$
\begin{aligned}
\widehat{P}^{(a)}(\lambda)=\frac{1}{\lambda} & \int_{0}^{\infty} d s_{0} e^{-\lambda s_{0}} F_{\lambda}\left(s_{0}\right) \sum_{n=0}^{\infty}(-1)^{n} \\
& \times \prod_{j=1}^{n}\left[\int_{0}^{\infty} d s_{j} e^{-\lambda s_{j}} G_{\lambda}\left(s_{0}+\sum_{l=1}^{j} s_{l}\right)\right],
\end{aligned}
$$

where

$$
\begin{aligned}
& F_{\lambda}(s)=\Delta^{2} \sin \pi \alpha \int_{0}^{\infty} d \tau e^{-\lambda \tau-S(\tau)} \sin \left\{\left[\epsilon_{0}+\epsilon(s)\right] \tau\right\} \\
& G_{\lambda}(s)=\Delta^{2} \cos \pi \alpha \int_{0}^{\infty} d \tau e^{-\lambda \tau-S(\tau)} \cos \left\{\left[\epsilon_{0}+\epsilon(s)\right] \tau\right\} .
\end{aligned}
$$

These functions are integrals over an effective intrablip interaction which is a function of both blip and sojourn lengths. As a result, the integrand of (3.4) no longer factorizes in the variables $s_{j}$, as it does in the case of 
constant bias where the sum is just a geometric series. The functions $F_{\lambda}(s)$ and $G_{\lambda}(s)$ can be directly related to a self-energy term $\hbar \Sigma$, where $\Sigma$ is given by

$$
\begin{aligned}
\Sigma(\lambda) & \equiv \Delta^{2} \cos (\pi \alpha) \int_{0}^{\infty} d \tau e^{-\lambda \tau} e^{-S(\tau)} \\
& =\Delta_{e}\left(\frac{\hbar \beta \Delta_{e}}{2 \pi}\right)^{1-2 \alpha} \frac{\Gamma(\alpha+\hbar \beta \lambda / 2 \pi)}{\Gamma(1-\alpha+\hbar \beta \lambda / 2 \pi)},
\end{aligned}
$$

and where $\Gamma(z)$ is the gamma function. In the second expression for $\Sigma(\lambda)$, we explicitly used the form (2.19) for the intrablip interaction $S(\tau)$, and we introduced the effective zero temperature tunneling splitting

$$
\Delta_{e}=\Delta\left(\frac{\Delta}{\omega_{c}}\right)^{\alpha /(1-\alpha)}[\cos (\pi \alpha) \Gamma(1-2 \alpha)]^{1 /(2-2 \alpha)}
$$

In terms of $\Sigma(\lambda)$, the functions $F_{\lambda}(\rho)$ and $G_{\lambda}(\rho)$ are given by

$$
\begin{aligned}
& F_{\lambda}(\rho)=-\frac{\tan \pi \alpha}{2 i}\left[\Sigma\left(\lambda_{+}\right)-\Sigma\left(\lambda_{-}\right)\right] \\
& G_{\lambda}(\rho)=\frac{1}{2}\left[\Sigma\left(\lambda_{+}\right)+\Sigma\left(\lambda_{-}\right)\right] \\
& \lambda_{ \pm}(\rho)=\lambda \pm i\left[\epsilon_{0}+\epsilon(\rho)\right] .
\end{aligned}
$$

By introducing $\rho_{j}=\sum_{l=0}^{j} s_{j}$ as new integration variables in (3.4), we can sum up the series for $\widehat{P}^{(a)}(\lambda)$ and obtain

$$
\begin{aligned}
\widehat{P}^{(a)}(\lambda)= & \int_{0}^{\infty} d \rho_{0} F_{\lambda}\left(\rho_{0}\right) e^{-\lambda \rho_{0}} \int_{0}^{\infty} d \rho_{1} e^{-\lambda \rho_{1}} \\
& \times \exp \left(-\int_{\rho_{0}}^{\rho_{0}+\rho_{1}} d \rho G_{\lambda}(\rho)\right) .
\end{aligned}
$$

Finally, noting that $F_{\lambda}(\rho)$ and $G_{\lambda}(\rho)$ are periodic functions of $\rho$ with period $2 \pi / \omega$, we can map the interval $[0, \infty]$ of the $\rho_{0}$ integral on the principal interval $[0,2 \pi / \omega]$. We then find

$$
\widehat{P}^{(a)}(\lambda)=\frac{K(\lambda)}{1-e^{-2 \pi \lambda / \omega}}
$$

where the function $K(\lambda)$ is given by

$$
\begin{aligned}
K(\lambda)= & \int_{0}^{2 \pi / \omega} d \rho_{0} F_{\lambda}\left(\rho_{0}\right) e^{-\lambda \rho_{0}} \int_{0}^{\infty} d \rho_{1} e^{-\lambda \rho_{1}} \\
& \times \exp \left(-\int_{\rho_{0}}^{\rho_{0}+\rho_{1}} d \rho G_{\lambda}(\rho)\right) .
\end{aligned}
$$

To show that $K(\lambda)$ does not have singularities for purely imaginary values of $\lambda$, we use again the periodicity property of the function $G_{\lambda}(\rho)$ to obtain

$$
\begin{aligned}
K(\lambda)= & \frac{1}{1-e^{-\left(2 \pi \lambda / \omega+J_{\lambda}\right)}} \int_{0}^{2 \pi / \omega} d \rho_{0} F_{\lambda}\left(\rho_{0}\right) e^{-\lambda \rho_{0}} \\
& \times \int_{0}^{2 \pi / \omega} d \rho_{1} e^{-\lambda \rho_{1}} \exp \left(-\int_{\rho_{0}}^{\rho_{0}+\rho_{1}} d \rho G_{\lambda}(\rho)\right)
\end{aligned}
$$

where

$$
J_{\lambda}=\int_{0}^{2 \pi / \omega} d \rho G_{\lambda}(\rho) .
$$

Now, the poles of $K(\lambda)$ are given by the solutions to the equation

$$
2 \pi \lambda / \omega+J_{\lambda}=2 n \pi i,
$$

where $n$ is an integer. It is clear that $\operatorname{Re} J_{\lambda}$ must be zero for purely imaginary solutions. But from the reflection formula of the $\Gamma$ function we get

$$
\begin{aligned}
\operatorname{Re} \Sigma(i \Omega)= & \frac{\Delta_{e}}{\pi}\left(\frac{\hbar \beta \Delta_{e}}{2 \pi}\right)^{1-2 \alpha}\left|\Gamma\left(\alpha+\frac{i \hbar \beta \Omega}{2 \pi}\right)\right|^{2} \\
& \times \sin (\pi \alpha) \cosh \left(\frac{\hbar \beta \Omega}{2 \pi}\right) .
\end{aligned}
$$

This is positive for any real $\Omega$, so that $\operatorname{Re} J_{\lambda}$ is nonzero for imaginary $\lambda$.

From this we see that, similar to the function $\mathcal{F}(\lambda, \omega)$ in (2.27), the poles of the function $K(\lambda)$ provide the transient dynamics of the system.

The stationary response of the system is given by the poles of the other factor in (3.9) lying on the imaginary axis at $\lambda=i n \omega$. Picking up all of them by contour integration, we finally get

$$
P^{(\mathrm{st})}(t)=\sum_{n=-\infty}^{+\infty} e^{-i n \omega t} P_{n}(\omega)
$$

where

$$
\begin{aligned}
P_{n}(\omega)= & \frac{\omega}{2 \pi} \int_{0}^{2 \pi / \omega} d \rho_{0} F_{-i n \omega}\left(\rho_{0}\right) e^{i n \omega \rho_{0}} \\
& \times \int_{0}^{\infty} d \rho_{1} e^{i n \omega \rho_{1}} \exp \left(-\int_{\rho_{0}}^{\rho_{0}+\rho_{1}} d \rho G_{-i n \omega}(\rho)\right) .
\end{aligned}
$$

Equation (3.13) together with (3.14) describes the steady-state behavior of $P^{(a)}(t)$ in the presence of an external periodic driving force of arbitrary strength in a parameter region characterized by $\hbar \omega \ll 2 \pi \alpha k_{B} T$ and within the validity range of the NIBA.

Using the periodicity of $F_{\lambda}(\rho)$ and $G_{\lambda}(\rho)$, it is again easy to show that a phase shift $\varphi$ in the driving force leads just to an identical phase shift in $P^{(\mathrm{st})}(t)$.

In the quasistatic limit $(\omega \rightarrow 0)$, the $P_{n}(\omega)$ are just the Fourier coefficients of $P^{(\text {equ })}\left[\epsilon_{0}+\epsilon(t)\right]$, where $P^{(\text {equ })}\left(\epsilon_{0}\right)=$ $\tanh \left(\frac{\hbar \beta \epsilon_{0}}{2}\right)$ is the equilibrium value $\lim _{t \rightarrow \infty} P^{(a)}(t)$ given by the NIBA in the case of constant bias $\epsilon_{0}[3,1]$.

In what follows, we will explicitly evaluate the Fourier coefficients $P_{n}(\omega)$ for a periodic driving with time dependence given by a cosine function and by a rectangular function. For monochromatic driving, the quantities $P_{ \pm 1}(\omega)$ are the only nonvanishing harmonics of $P^{(a)}(t)$ in the linear response regime. For large amplitude $\hat{\epsilon}$ of the monochromatic driving force, also higher harmonics $P_{n}(\omega)$ with $|n|>1$ may become important. However, in a wide range of experimental setups, e.g., when lock-in techniques are used, or when the system of interest is coupled to a high-quality macroscopic oscillator such as a resonant cavity or a vibrating reed, the response function $P_{1}(\omega)$ is the most relevant quantity even for large $\hat{\epsilon}$. Therefore we will focus our attention on $P_{1}(\omega)$. It is 
immediately obvious from (2.31) that the function

$$
\tilde{\chi}^{(\mathrm{nl})}(\omega ; \hat{\epsilon}) \equiv \frac{P_{1}(\omega)}{\hbar \hat{\epsilon}}
$$

is a suitable quantity which generalizes the dynamical susceptibility to the nonlinear response regime. Clearly, for $\hat{\epsilon} \rightarrow 0$, the function $\widetilde{\chi}^{(\mathrm{nl})}(\omega ; \hat{\epsilon})$ reduces to the linear dynamical susceptibility $\widetilde{\chi}(\omega)$ discussed in Sec. II B. Within the approximations stated above, we now arrive at an explicit expression for $\tilde{\chi}^{(\mathrm{nl})}(\omega ; \hat{\epsilon})$.

From Eq. (3.14) we find for the response function

$$
\begin{aligned}
P_{1}(\omega)= & \frac{\omega}{2 \pi} \int_{0}^{2 \pi / \omega} d \rho_{0} F_{0}\left(\rho_{0}\right) e^{i \omega \rho_{0}} \int_{0}^{\infty} d \rho_{1} e^{i \omega \rho_{1}} \\
& \times \exp \left(-\int_{\rho_{0}}^{\rho_{0}+\rho_{1}} d \rho G_{0}(\rho)\right)
\end{aligned}
$$

In accordance with the condition $\omega \ll 2 \pi \alpha / \hbar \beta$, here we dropped the frequency dependence in the functions $F$ and $G$.

Let us now first consider the case of the "rectangular shape" of the driving

$$
\epsilon(t)=\hat{\epsilon} \operatorname{sgn}(\cos \omega t) \text {. }
$$

This case is of particular interest because it leads to analytic results in compact form which will show that relaxation peaks are indeed to be found in the parameter range that we chose. With the expression (3.17) for $\epsilon(t)$, the functions $F_{\lambda=0}(\rho)$ and $G_{\lambda=0}(\rho)$ in (3.16) jump abruptly between the values $F_{+}$and $F_{-}$, and $G_{+}$and $G_{-}$, respectively, where

$$
\begin{aligned}
& F_{ \pm}=-\frac{\tan \pi \alpha}{2 i}\left[\Sigma\left(i\left(\epsilon_{0} \pm \hat{\epsilon}\right)\right)-\Sigma\left(-i\left(\epsilon_{0} \pm \hat{\epsilon}\right)\right)\right] \\
& G_{ \pm}=\frac{1}{2}\left[\Sigma\left(i\left(\epsilon_{0} \pm \hat{\epsilon}\right)\right)+\Sigma\left(-i\left(\epsilon_{0} \pm \hat{\epsilon}\right)\right)\right] .
\end{aligned}
$$

With this simplification, it is only slightly tedious but straightforward to evaluate the expression (3.16) in analytic form. This can be done by splitting the integration domains into intervals where $\epsilon(\rho)$ is constant and by performing a summation afterwards. We then find

$$
P_{1}(\omega)=\mathcal{A}\left[\frac{\Gamma_{+}}{\Gamma_{+}-i \omega} \mathcal{C}_{+}(\omega)+\frac{\Gamma_{-}}{\Gamma_{-}-i \omega} \mathcal{C}_{-}(\omega)\right]
$$

where the rates $\Gamma_{ \pm}$, the amplitude $\mathcal{A}$, and the form factors $\mathcal{C}_{ \pm}(\omega)$ are given by

$$
\begin{aligned}
\Gamma_{ \pm} & =G_{ \pm} \\
\mathcal{A} & =\frac{1}{2 \pi}\left(\frac{F_{+}}{G_{+}}-\frac{F_{-}}{G_{-}}\right), \\
\mathcal{C}_{ \pm}(\omega) & =1 \mp \frac{i \omega}{\Gamma_{ \pm}} \frac{\sinh \left(\frac{\pi\left(\Gamma_{+}-\Gamma_{-}\right)}{2 \omega}\right)}{\sinh \left(\frac{\pi\left(\Gamma_{+}+\Gamma_{-}\right)}{2 \omega}\right)}
\end{aligned}
$$

Equation (3.18) describes a superposition of two quasielastic resonances around $\omega=0$ with widths $\Gamma_{+}$ and $\Gamma_{-}$, respectively. Note that the expression (3.18) is well defined in the limit $\omega \rightarrow 0$ since $\mathcal{C}_{ \pm}(\omega \rightarrow 0)=1$. The form (3.18) can be simplified further in the cases where the rates $\Gamma_{+}$and $\Gamma_{-}$are either roughly equal or of different magnitude.

The case $\Gamma_{+} \approx \Gamma_{-}$applies either for $\left|\epsilon_{0}\right| \gg \hat{\epsilon}$ or for $\left|\epsilon_{0}\right| \ll \hat{\epsilon}$. Then the response function simplifies further to the form

$$
P_{1}(\omega) \approx 2 \mathcal{A} \frac{\Gamma_{+}}{\Gamma_{+}-i \omega} .
$$

On the other hand, for $\Gamma_{+} \ll \Gamma_{-}$, we find as leading terms

$$
\operatorname{Re} P_{1}(\omega)=\mathcal{A} \times \begin{cases}1 & \left(\omega \ll \Gamma_{+}\right) \\ \frac{\pi \Gamma_{+}}{\omega} & \left(\Gamma_{+} \ll \omega \ll \Gamma_{-}\right) \\ \frac{2 \Gamma_{+} \Gamma_{-}}{\omega^{2}} & \left(\omega \gg \Gamma_{-}\right),\end{cases}
$$

and

$\operatorname{Im} P_{1}(\omega)=\mathcal{A} \times\left\{\begin{array}{cl}\frac{\omega}{\Gamma_{+}} & \left(\omega \ll \Gamma_{+}\right) \\ \frac{2 \Gamma_{+}}{\omega} & \left(\Gamma_{+} \ll \omega \ll \Gamma_{-}\right) \\ \frac{4 \Gamma_{+}}{\omega} & \left(\omega \gg \Gamma_{-}\right),\end{array}\right.$

which shows quite simple algebraic dependence on $\omega$. In each case, both the real and the imaginary part of $P_{1}(\omega)$ decay at least as $\omega^{-1}$ for $\omega \gg \Gamma_{+}$, i.e., $\Gamma_{+}$is always the relevant frequency scale of the incoherent dynamics.

For weak damping $\alpha \ll 1$, we find for the widths

$$
\Gamma_{ \pm}=\Delta_{e}^{2} \frac{\pi \alpha\left(\epsilon_{0} \pm \hat{\epsilon}\right)}{\left(2 \pi \alpha k_{B} T / \hbar\right)^{2}+\left(\epsilon_{0} \pm \hat{\epsilon}\right)^{2}} \operatorname{coth}\left(\frac{\hbar\left(\epsilon_{0} \pm \hat{\epsilon}\right)}{2 k_{B} T}\right)
$$

as follows from (3.5), (3.7), and (3.19). In this case, the condition $\hbar \Gamma_{+} \ll 2 \pi \alpha k_{B} T$ is equivalent to

$$
\epsilon_{0}+\hat{\epsilon} \gg \max \left(\Delta_{e}, \hbar \Delta_{e}^{2} / 2 k_{B} T\right) .
$$

Hence our initially chosen parameter range $\hbar \omega \ll$ $2 \pi \alpha k_{B} T$ contains the main features of relaxation for any TLS with strong enough intrinsic or external bias. In particular, this means that the saturation limit $\hat{\epsilon} \rightarrow \infty$ can be treated by our method for any set of parameters.

Figures 1 and 2 show the real and imaginary parts of the response function $P_{1}(\omega)$ in a double-logarithmic plot for values of $\hat{\epsilon}$ extending from the linear response regime to the saturation limit. Here, and in the subsequent figures, the parameters $\omega, \epsilon_{0}, \hat{\epsilon}$, and $k_{B} T / \hbar$ are scaled by the effective tunneling matrix element $\Delta_{e}$. The line shape is the same for both linear response and saturation, as suggested by the structure of (3.22). The curves correspond to Lorentzian line shapes, and the straight line sections are the characteristics of the power laws on the slopes of the peak.

Next, consider the behavior of the response function $P_{1}(\omega)$ for a harmonic driving force

$$
\epsilon(t)=\hat{\epsilon} \cos (\omega t) .
$$

Now, the functions $F$ and $G$ in (3.16) depend on the con- 


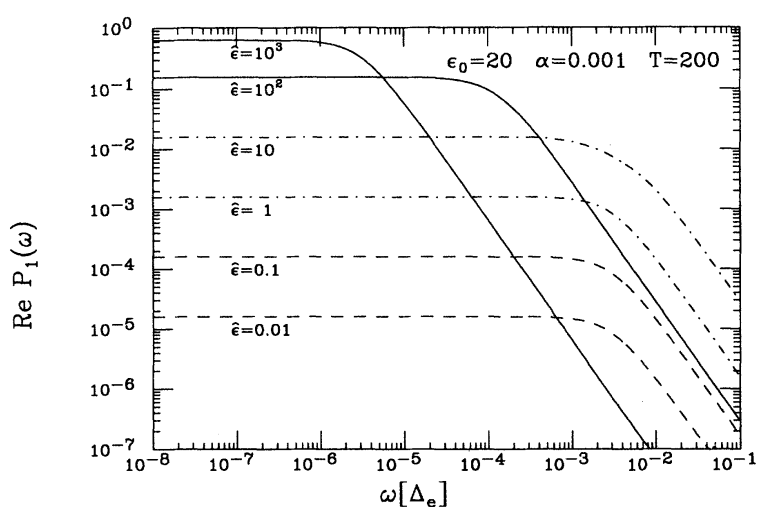

FIG. 1. Real part of the response function $P_{1}(\omega)$ for different amplitudes $\hat{\epsilon}$ of a driving signal of rectangular shape at low frequencies. For values of $\hat{\epsilon}$ ranging from the linear response limit to the saturation regime, the shape of the curves remains a simple Lorentzian centered around zero (with width and height depending on $\hat{\epsilon}$ ). The parameter $\alpha$ is a dimensionless coupling constant of the dissipative system-environment coupling. Frequencies are given in units of $\Delta_{e}$, temperature in units of $\hbar \Delta_{e} / k_{B}$.

tinuously varying bias $\epsilon_{0}+\epsilon(t)$. With the form (3.27), it is no longer as easy (as it was in the rectangular case where we had only two possible values for $F$ and $G$ ) to evaluate $P_{1}(\omega)$ analytically, and we have to resort to numerical computation. For weak damping we now have

$$
\begin{aligned}
F_{0}(\rho)= & \pi \alpha \Delta_{e}^{2} \frac{\epsilon_{0}+\epsilon(\rho)}{\left(2 \pi \alpha k_{B} T / \hbar\right)^{2}+\left[\epsilon_{0}+\epsilon(\rho)\right]^{2}} \\
G_{0}(\rho)= & \pi \alpha \Delta_{e}^{2} \frac{\epsilon_{0}+\epsilon(\rho)}{\left(2 \pi \alpha k_{B} T / \hbar\right)^{2}+\left[\epsilon_{0}+\epsilon(\rho)\right]^{2}} \\
& \times \operatorname{coth}\left(\frac{\hbar\left[\epsilon_{0}+\epsilon(\rho)\right]}{2 k_{B} T}\right) .
\end{aligned}
$$

Inserting these expressions into (3.16), the remaining integrals can be computed only numerically.

Figures 3 and 4 show some results of this evaluation.

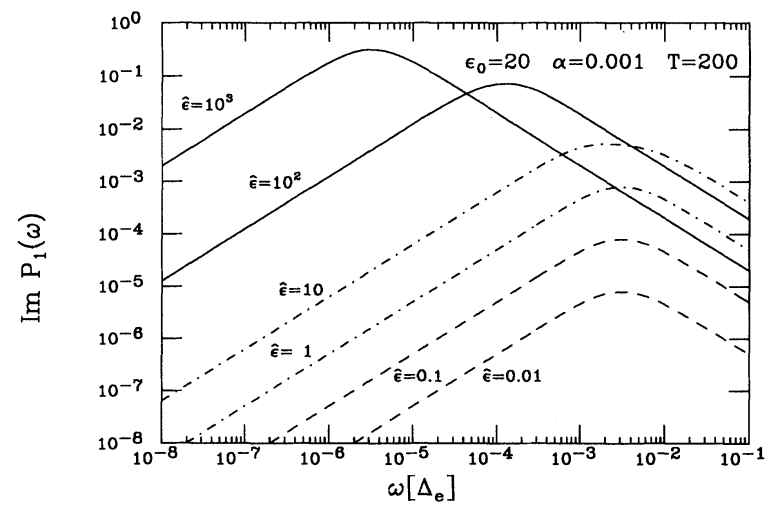

FIG. 2. Imaginary part of the response function $P_{1}(\omega)$ for different amplitudes $\hat{\epsilon}$ of a driving signal of rectangular shape at low frequencies. The curves correspond to Lorentzians. Same parameters as in Fig. 1.

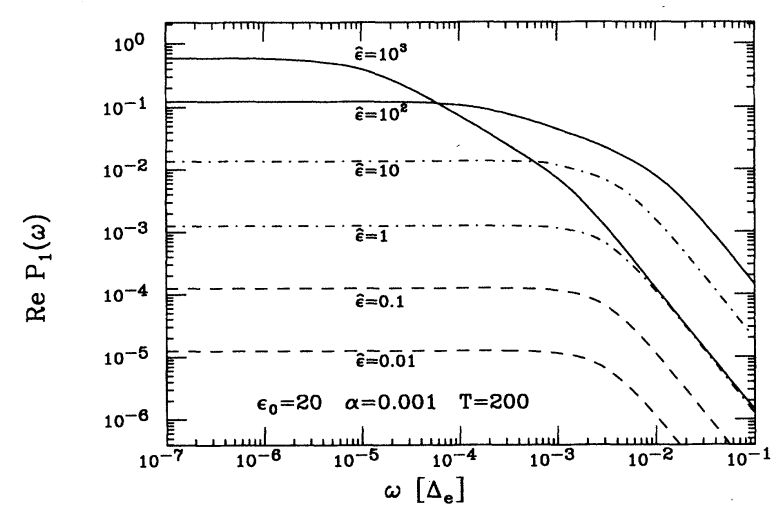

FIG. 3. Real part of the response function $P_{1}(\omega)$ for different amplitudes $\hat{\epsilon}$ of low-frequency external driving with a sinusoidal time dependence. Strong deviations from a Lorentzian line shape are observed in a wide intermediate frequency range for any values of $\hat{\epsilon}$ above the linear response regime. Only at frequencies below the minimum relaxation rate $\Gamma_{+}$is the nonlinear response comparable to the rectangular case.

We plotted $\operatorname{Im} P_{1}(\omega)$ and $\operatorname{Re} P_{1}(\omega)$ versus $\omega$ in a doublelogarithmic scale for different values of $\hat{\epsilon}$ for parameters in the region of moderately high temperatures and weak damping $\left(k_{B} T \gg \hbar \Delta, \alpha \ll 1\right)$ with fixed $\epsilon_{0}$. There is a rough similarity to the case of the rectangular driving function, but there are also some striking differences.

The function $\operatorname{Im} P_{1}(\omega)$ shows a relaxation peak centered around an effective relaxation rate which is larger than $\Gamma_{+}$. With growing $\hat{\epsilon}$, this rate decreases while the value of the maximum increases and reaches a saturation value. As in our results for a "rectangular shape" of the driving force, the asymptotic decay of the response function $P_{1}(\omega)$ is visible already within the range $\omega \ll 2 \pi \alpha k_{B} T / \hbar$. The major difference between the two types of driving is the fact that strong deviations from the Lorentzian line shape are observed in the saturation regime for harmonic driving. The intermediate-frequency range between the straight sections of the curves can extend over several powers of 10 , indicating a wide range

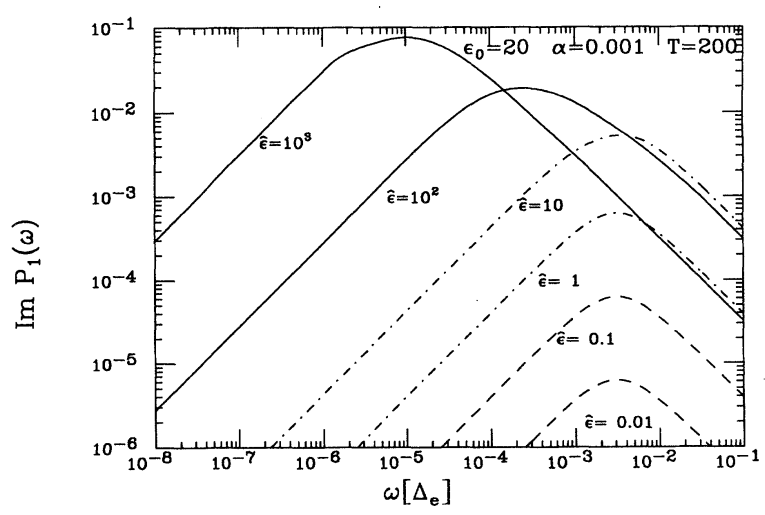

FIG. 4. Imaginary part of the response function $P_{1}(\omega)$ for different amplitudes $\hat{\epsilon}$ of low-frequency external driving with a sinusoidal time dependence. See Fig. 3 for comments. 
of time scales for relaxation even for a single two-level system with fixed parameters.

\section{THE CASE $\alpha=\frac{1}{2}$}

For the special value $\frac{1}{2}$ of the Ohmic damping strength $\alpha$, the exact formal expression for $P^{(a)}(t)$ can be summed exactly. At first glance, the series expression (2.21) or (2.24) seems ill defined due to the multiplicity of $\cos (\pi \alpha)$ factors, which become zeros for $\alpha=\frac{1}{2}$, but is well defined for $\alpha$ arbitrarily close to $\frac{1}{2}$. Taking the limit $\alpha \rightarrow \frac{1}{2}$ actually simplifies the calculation considerably since the multiple zeros at $\alpha=\frac{1}{2}$ need to be offset by a corresponding number of divergences in the integrals over the blip and sojourn lengths in order to render nonvanishing contributions.

Consider the integral over the interval between two neighboring attractive flips

$B(\alpha) \equiv \Delta^{2} \int_{0}^{\infty} d \tau \cos (\pi \alpha) f(\tau, \alpha)\left(\frac{\hbar \beta \omega_{c}}{\pi} \sinh \frac{\pi \tau}{\hbar \beta}\right)^{-2 \alpha}$

where the function $f(\tau, \alpha)$, which contains the interactions with all the other flips, is bounded. It is to be noted that the integrand is uniformly bounded on the interval $\left[\tau_{0}, \infty\right]$ for any positive parameter $\tau_{0}$, and it vanishes in the limit $\alpha \rightarrow \frac{1}{2}$. So the only contribution to (4.1) in this limit comes from the interval $\left[0, \tau_{0}\right]$, where $\tau_{0}$ can be arbitrarily small. Then $f(\tau, \alpha)$ can be replaced by $f(0, \alpha)$, and expression (4.1) is transformed into

$$
\begin{aligned}
B\left(\alpha=\frac{1}{2}\right) & =\frac{\Delta^{2}}{\omega_{c}} \lim _{\alpha \rightarrow 1 / 2} f(0, \alpha) \cos (\pi \alpha) \Gamma(1-2 \alpha) \\
& =\Gamma f\left(0, \frac{1}{2}\right)
\end{aligned}
$$

where

$$
\Gamma \equiv \Delta_{e}\left(\alpha=\frac{1}{2}\right)=\frac{\pi}{2} \frac{\Delta^{2}}{\omega_{c}}
$$

is the natural frequency scale of the system for $\alpha=\frac{1}{2}$. If the integration over a blip or sojourn length is handled in this way, we shall allude to a "collapsed" blip or sojourn. The technique of collapsed blips or sojourns used in evaluating this limit has been described before by two of the authors [19] in the case of a static bias. Here we generalize the discussion to a time-periodic driving force.

As $\hat{P}^{(a)}(\lambda)$ contains a factor $(\cos \pi \alpha)^{n-1}$ in order $\Delta^{2 n}$, $n-1$ blips and sojourns are collapsed. The interactions of any collapsed blip or sojourn with other blips vanish, and the only remaining interaction is between the two flips forth and back that are neither part of a collapsed blip nor of a collapsed sojourn. These two instantons form a so-called "extended" blip. This generalized blip is always the first one, and it may contain inside an arbitrary number of collapsed sojourns. The extended blip is followed by any number of collapsed blips. Thus $\hat{P}^{(a)}(\lambda)$ takes the form

$$
\begin{aligned}
\hat{P}^{(a)}(\lambda)= & \Delta^{2} \sum_{l=1}^{\infty} \sum_{m=1}^{\infty}(-1)^{l+m} \Gamma^{l+m-2} 2^{1-l} \\
& \times \int_{0}^{\infty} d s_{0} e^{-\lambda s_{0}} \int_{0}^{\infty} d^{m} s e^{-\lambda \sum_{j=1}^{m} s_{j}} \\
& \times \int_{0}^{\infty} d^{l} \tau e^{-\lambda \sum_{j=1}^{l} \tau_{j}} \exp \left[-S\left(\sum_{j=1}^{l} \tau_{j}\right)\right] \\
& \times \sin \left[\epsilon_{0} \sum_{j=1}^{l} \tau_{j}+g\left(s_{0}+\sum_{j=1}^{l} \tau_{j}\right)-g\left(s_{0}\right)\right] .
\end{aligned}
$$

Here, $l-1$ is the number of collapsed sojourns inside the extended blip, and $m-1$ is the number of succeeding collapsed blips. The integrals over the blip times $\tau_{j}$ inside the extended blip can easily be rewritten as a single breathing mode integral of the extended blip, and the integrations over sojourn times after the extended blip are now trivial. So only two integrations remain,

$$
\begin{aligned}
\hat{P}^{(a)}(\lambda)= & \frac{\Delta^{2}}{\lambda} \frac{1}{(1+\Gamma / \lambda)} \\
& \times \int_{0}^{\infty} d s e^{-\lambda s} \int_{0}^{\infty} d \tau e^{-\lambda \tau-\Gamma \tau / 2-S(\tau)} \\
& \times \sin \left[\epsilon_{0} \tau+g(s+\tau)-g(s)\right]
\end{aligned}
$$

Here, the factor $e^{-\Gamma \tau / 2}$ of the integrand arises from the noninteracting gas of collapsed sojourns inside the extended blip, while the factor $\frac{1}{1+\Gamma / \lambda}$ is due to the noninteracting gas of collapsed blips succeeding the extended one. Apart from the kernel $e^{-\lambda s}$ of the Laplace transformation, the integrand is a periodic function of $s$ and can be given as a Fourier series, which leads to an expression of the form

$$
\begin{aligned}
& \hat{P}^{(a)}(\lambda) \\
& \quad=\frac{\Delta^{2}}{\lambda+\Gamma} \sum_{n=0}^{\infty} \frac{1}{\lambda+i n \omega} \int_{0}^{\infty} d \tau e^{-\lambda \tau-\Gamma \tau / 2-S(\tau)} F_{n}(\tau) .
\end{aligned}
$$

For harmonic driving $\epsilon(t)=\hat{\epsilon} \cos (\omega t-\varphi)$, which is the case of most interest, the coefficients $F_{n}(\tau)$ can be given explicitly [with $J_{n}(z)$ denoting the Bessel function of the first kind],

$$
\begin{aligned}
F_{2 k}(\tau)= & (-1)^{k} e^{i 2 k \varphi} e^{-i k \omega \tau} \sin \left(\epsilon_{0} \tau\right) J_{2 k}\left(\frac{2 \hat{\epsilon}}{\omega} \sin \frac{\omega \tau}{2}\right) \\
F_{2 k+1}(\tau)= & (-1)^{k} e^{i(2 k+1) \varphi} e^{-i\left(k+\frac{1}{2}\right) \omega \tau} \cos \left(\epsilon_{0} \tau\right) \\
& \times J_{2 k+1}\left(\frac{2 \hat{\epsilon}}{\omega} \sin \frac{\omega \tau}{2}\right) .
\end{aligned}
$$

The integral on the right-hand side of (4.4) is the Laplace transform of a function that is bounded by a decaying exponential function. Thus the only singularities on the imaginary axes are again simple poles at $\lambda=-i n \omega$. It is these poles that determine the periodic long-time behavior of $P^{(a)}(t)$, and from their residues we extract the Fourier coefficients $P_{n}$ of 


$$
P^{(\mathrm{st})}(t)=\sum_{n=-\infty}^{\infty} e^{-i n \omega t} P_{n}(\omega)
$$

We finally arrive at the form

$$
P_{n}(\omega)=\frac{\Gamma}{\Gamma-i n \omega} \frac{2}{\hbar \beta} \int_{0}^{\infty} d \tau \frac{e^{(i n \omega-\Gamma / 2) \tau}}{\sinh \frac{\pi \tau}{\hbar \beta}} F_{n}(\tau)
$$

The remaining integral can be evaluated analytically only in special parameter regions. Note that $P_{n}$ depends on $\varphi$ only through a phase factor $e^{i n \varphi}$, i.e., $\varphi$ enters again $P^{(\text {st })}(t)$ only as a trivial phase shift. So we can set again $\varphi=0$ in the following without any loss of generality.

Inserting the limiting forms of the Bessel functions for small arguments into the functions $F_{n}(\tau)$, we see that $P_{n}$ behaves as $\hat{\epsilon}^{|n|}$ for small $\hat{\epsilon}$. Thus in leading order $P_{0}$ becomes independent of $\hat{\epsilon}$ and $\omega$, and coincides with the equilibrium value $\left\langle\sigma_{z}\right\rangle_{\beta} \equiv P^{(\text {equ })}=\lim _{t \rightarrow \infty} P^{(a)}(t)$ for constant bias. We find

$$
P_{0} \equiv P^{(\text {equ })}=\frac{2}{\pi} \operatorname{Im} \psi\left(x_{+}\right)
$$

where $\psi(z)$ is the digamma function, and

$$
x_{ \pm}=\frac{1}{2}+\frac{\hbar \beta}{2 \pi}\left(\frac{\Gamma}{2} \pm i \epsilon_{0}\right) .
$$

The linear response regime is fully determined by $P_{ \pm 1}(\omega)$. Using the relation

$$
P_{ \pm 1}(\omega)=\hbar \hat{\epsilon} \tilde{\chi}( \pm \omega)
$$

we find from (4.6) that the linear dynamical susceptibility takes the analytic form

$$
\begin{aligned}
\tilde{\chi}(\omega)= & \frac{1}{\hbar \omega} \frac{\Gamma}{(\Gamma-i \omega)} \frac{1}{2 \pi i} \phi(\omega) \\
\phi(\omega)= & \psi\left(x_{+}\right)-\psi\left(x_{+}-i \frac{\hbar \beta \omega}{2 \pi}\right) \\
& +\psi\left(x_{-}\right)-\psi\left(x_{-}-i \frac{\hbar \beta \omega}{2 \pi}\right) .
\end{aligned}
$$

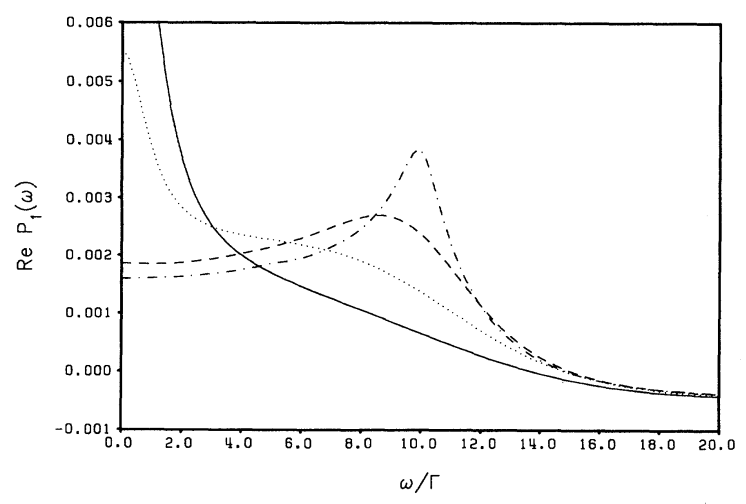

FIG. 5. Real part of the response function $P_{1}(\omega)$ for strong dissipation $\left(\alpha=\frac{1}{2}\right)$ with an intrinsic asymmetry $\epsilon_{0}=10 \Gamma$, a moderate amplitude $\hat{\epsilon}=\Gamma$ of the external (sinusoidally varying) asymmetry, and the temperatures $k_{B} T=3 \hbar \Gamma$ (fully drawn), $k_{B} T=2 \hbar \Gamma$ (dotted), $k_{B} T=\hbar \Gamma$ (dashed), and $T=0$ (dashed-dotted). At low temperature, a resonance near $\omega=\epsilon_{0}$ is observed.

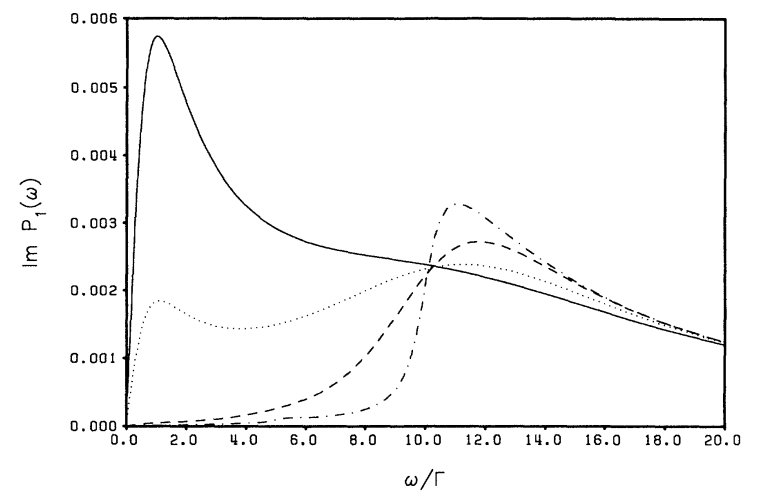

FIG. 6. Imaginary part of the response function $P_{1}(\omega)$ for strong dissipation at moderate amplitude. Comments of Fig. 5 apply.

The opposite, extreme nonlinear response limit $\hat{\epsilon} \rightarrow \infty$ leads to a remarkably simple analytic result. Here, the $P_{n}$ reach saturation values that are also independent of the intrinsic bias $\epsilon_{0}$ and of temperature,

$$
\lim _{\hat{\epsilon} \rightarrow \infty} P_{n}(\omega)=\left\{\begin{array}{lc}
0, & n \text { even } \\
\frac{2}{\pi|n|} \frac{\Gamma}{\Gamma-i n \omega}, & n \text { odd } .
\end{array}\right.
$$

The expression (4.6) for the response function $P_{1}(\omega)$ has been evaluated numerically as a function of the parameters $\epsilon_{0}, \hat{\epsilon}, k_{B} T / \hbar$, and $\omega$. For high temperature or large bias amplitude $\hat{\epsilon}$, the function $P_{1}(\omega)$ displays just a simple relaxation peak of width $\Gamma$.

Figures 5-8 are representatives of the different kinds of qualitative behavior found in our investigation. Here we use $\Gamma$ as the frequency scale. At moderate values of the

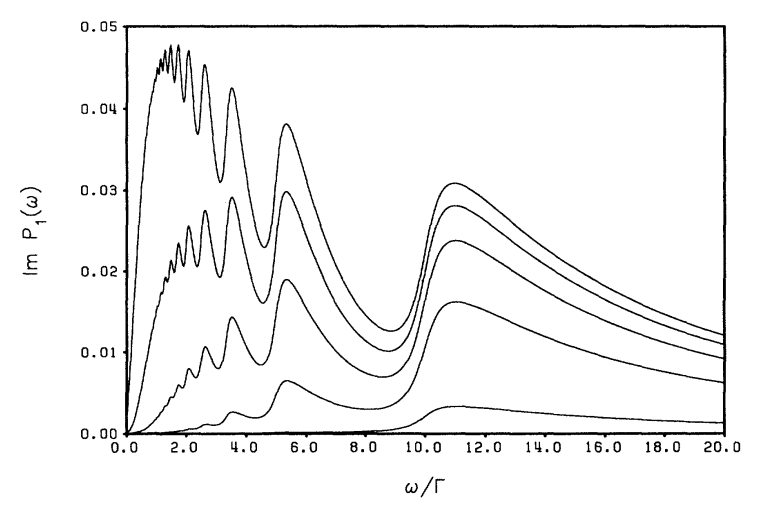

FIG. 7. Response function $P_{1}(\omega)$ (imaginary part) with strong dissipation for different bias amplitudes in the nonlinear regime. Resonances are now also found near submultiples of $\epsilon_{0}$. These are to be attributed to processes involving multiple quanta of the environmental field (e.g., phonons, for tunneling systems in solids). The total response function is a superposition of these resonances and a relaxation peak which grows with increasing $\hat{\epsilon}$. The parameters are $\alpha=\frac{1}{2}$, $k_{B} T=0.05 \hbar \Gamma, \epsilon_{0}=10 \Gamma$, and $\hat{\epsilon}=\Gamma, 5 \Gamma, 7.5 \Gamma, 9 \Gamma$, and $10 \Gamma$ (bottom to top). 


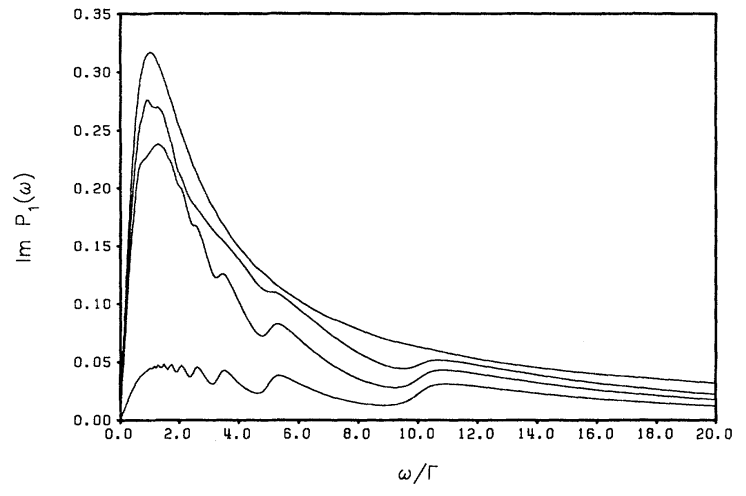

FIG. 8. The transition to incoherent dynamics for a very strong external force becomes apparent in this plot of $\operatorname{Im} P_{1}(\omega)$. The parameters are $\alpha=\frac{1}{2}, k_{B} T=0.05 \hbar \Gamma$, $\epsilon_{0}=10 \Gamma$, and $\hat{\epsilon}=10 \Gamma, 15 \Gamma, 20 \Gamma$, and $100 \Gamma$ (bottom to top). For $\hat{\epsilon}=100 \Gamma$ the deviations from the asymptotic result (4.11) are already barely perceptible in the plot.

amplitude $\hat{\epsilon}$ (Figs. 5 and 6 ) a relaxation peak and a single resonance peak near $\epsilon_{0}$ are observed, whose heights vary strongly with temperature.

Beyond the linear response limit, resonances appear also at submultiples of the main resonance frequency due to the generation of higher harmonics (Fig. 7). In addition, a relaxation peak is clearly visible although the temperature is very low.

For $\hat{\epsilon}>\epsilon_{0}$ (Fig. 8), there are no well-defined resonances, rather an erratic pattern of maxima and minima emerges. A further increase of $\hat{\epsilon}$ (keeping all other parameters fixed) leads to the simple result (4.11). This case is in fact a good test of the numerical integration algorithm employed.

\section{CONCLUSION}

In this paper, we have studied the dissipative two-state system for Ohmic friction. It may be viewed, e.g., as an archetypal model for translational tunneling systems in a metallic environment. Using a real-time functional integral description, we found exact formal expressions describing the nonlinear response for periodic driving in the steady state. The solutions were in the form of a series in the number of the system's transitions. In the linear response regime, we followed two different lines of thinking and we showed that the resulting expressions for the dynamical susceptibility are identical.

In the region of incoherent tunneling, we summed the series expression in analytic form and discussed the steady-state behavior for two different shapes of the periodic driving force. We found strikingly different behaviors of the line shapes in the saturation regime. For harmonic driving, the results indicate a wide range of time scales of relaxation even for a single TLS with fixed parameters.

For a special value of the Ohmic viscosity we obtained the solution in analytic form (apart from quadratures) for arbitrary temperature and driving parameters. The nonlinear dynamical susceptibility shows a rich variety of behaviors under variation of the parameters.

A discussion of the changes in the acoustic properties of amorphous metals which are due to the effects of the nonlinear susceptibility will be given elsewhere.

\section{ACKNOWLEDGMENTS}

This report was supported in part by the Deutsche Forschungsgemeinschaft (DFG, Bonn) under Contract No. We 1124-2/3 and in part by the SCIENCE program of the European Community under Contract No. SC1*-CT91-0656 (TSTS).
[1] For a general discussion of open quantum systems including a variety of applications, the general methods, archetypal models, and main features, see, e.g., U. Weiss, Quantum Dissipative Systems, Series in Modern Condensed Matter Physics Vol. 2 (World Scientific, Singapore, 1993).

[2] A. O. Caldeira and A. J. Leggett, Phys. Rev. Lett. 46, 211 (1981); Ann. Phys. (N.Y.) 149, 374 (1983); 153, 445(E) (1983).

[3] A. J. Leggett, S. Chakravarty, A. T. Dorsey, M. P. A. Fisher, A. Garg, and W. Zwerger, Rev. Mod. Phys. 59, 1 (1987).

[4] S. Chakravarty and S. Kivelson, Phys. Rev. Lett. 50, 1811 (1983).

[5] M. H. Devoret, D. Estève, J. M. Martinis, A. Cleland, and J. Clarke, Phys. Rev. B 36, 58 (1987).

[6] J. E. Combariza, B. Just, J. Manz, and G. K. Paramonov, J. Phys. Chem. 95, 10351 (1992).

[7] R. Bavli and H. Metiu, Phys. Rev. Lett. 69, 1986 (1992).

[8] L. M. Sander and H. B. Shore, Phys. Rev. B 3, 1472 (1971).

[9] S. Chakravarty, Phys. Rev. Lett. 49, 681 (1982); A. J.
Bray and M. A. Moore, ibid. 49, 1546 (1982); V. Hakim, A. Muramatsu, and F. Guinea, Phys. Rev. B 30, 464 (1984); A. Schmid, Phys. Rev. Lett. 51, 1506 (1983); S. Bulgadaev, Pis'ma Zh. Eksp. Teor. Phys. 39, 264 (1984) [JETP Lett. 39, 314 (1985)]; F. Guinea, V. Hakim, and A. Muramatsu, Phys. Rev. Lett. 54, 263 (1985).

[10] F. Grossmann, P. Jung, T. Dittrich, and P. Hänggi, Z. Phys. B 84, 315 (1991); F. Grossmann, T. Dittrich, P. Jung, and P. Hänggi, Phys. Rev. Lett. 67, 516 (1991); F. Grossmann and P. Hänggi, Europhys. Lett. 18, 571 (1992); T. Dittrich, B. Oelschlägel, and P. Hänggi, ibid. 22, 5 (1993).

[11] J. M. Gomez Llorente and J. Plata, Phys. Rev. A 45, 6958 (1992).

[12] P. Esquinazi, R. König, F. Pobell, F. Dietzel, G. Weiss, and S. Hunklinger, in Phonons 89 , edited by S. Hunklinger, W. Ludwig, and G. Weiss (World Scientific, Singapore, 1990).

[13] P. Esquinazi, R. König, and F. Pobell, Z. Phys. B 87, 305 (1992).

[14] For a review of the standard tunneling model for metallic glasses, see J. L. Black, in Glassy Metals I, edited by H.- 
J. Güntherodt and H. Beck, Topics in Applied Physics Vol. 46 (Springer, Berlin, 1981), Chap. 8.

[15] A. K. Raychaudhuri and S. Hunklinger, Z. Phys. B 57, 113 (1984); P. Esquinazi, H. M. Ritter, H. Neckel, G. Weiss, and S. Hunklinger, ibid. 64, 81 (1986).

[16] J. Stockburger, U. Weiss, and R. Görlich, Z. Phys. B 84, 457 (1991).
[17] J. Kondo, in Fermi Surface Effects, edited by J. Kondo and A. Yoshimori, Springer Series in Solid-State Sciences, Vol. 77 (Springer, Berlin, 1988).

[18] R. P. Feynman and F. L. Vernon, Ann. Phys. (N.Y.) 24, 118 (1963).

[19] M. Sassetti and U. Weiss, Phys. Rev. A 41, 5383 (1990). 